\title{
A novel approach to analysis of phage clones by reference strand mediated conformation analysis
}

\author{
Declan P. Prendergast ${ }^{\mathrm{a}, *}$, M. Isla Halliday ${ }^{\mathrm{a}, * *}$, \\ Neil V. McFerran ${ }^{\mathrm{a}}$, Martin Curran ${ }^{\mathrm{b}}$, \\ Brian McIhatton ${ }^{\mathrm{b}}$, Derek Middleton ${ }^{\mathrm{b}}$, \\ Mark T. Fox ${ }^{\mathrm{a}}$ and Andrew Wallace ${ }^{\mathrm{a}}$ \\ ${ }^{\mathrm{a}}$ Centre for Peptide and Protein Engineering, The \\ Queen's University of Belfast, Medical Biology \\ Centre, 97 Lisburn Road, Belfast, BT9 7BL, N. Ireland \\ ${ }^{\mathrm{b}}$ Northern Ireland Histocompatability and \\ Immunogenetics Laboratory, Blood Transfusion \\ Building, Belfast City Hospital, Lisburn Road, Belfast, \\ BT9 7TS, N. Ireland
}

\section{Introduction}

The use of phage display peptide libraries has developed into a very useful technology for the discovery of novel peptide ligands that bind to diverse targets. Such libraries comprise vast collections of short, randomised peptide sequences from which phage with desirable binding activities can be selected by a process of affinity purification termed "biopanning". However, this selection can result in over-representation of individual clones in a successful panning, leading to costly and time-consuming DNA sequencing of identical clones. In this study, we examined phage clones by reference strand mediated conformation analysis (RSCA) with a view to avoiding repetitive sequencing of multiply represented identical clones arising from the panning procedure. In addition, we investigated RSCA's potential to facilitate the identification of multiple incidences of the same sequence as an indicator of clonal enrichment by a successful panning procedure.

*Corresponding author: Tel.: +44 28 90272262; Fax: +44 28 90236505; E-mail: d.prendergast@qub.ac.uk.

** Deceased 16 July 1999.

\section{Methods}

\subsection{Biopanning procedure}

Phage were subjected to a biopanning procedure in solution. Briefly, $10^{11}$ phage of a disulphide constrained 9-mer peptide library were incubated with $2.7 \mu \mathrm{g}$ anti-ferritin $\mathrm{H} 107$ primary Ab overnight (o/n) at $4^{\circ} \mathrm{C}$. The mixture was reacted with $1.35 \mu \mathrm{g}$ biotinylated anti-mouse secondary $\mathrm{Ab}$ for $4 \mathrm{~h}$ room temperature (rt), diluted with TBS- $0.5 \%$ Tween 20 and aliquots transferred to streptavidin coated microtitre wells. After rocking for $10 \mathrm{~min}$ at $\mathrm{rt}$, phage were removed and plates washed every 5 min with TBS- $0.5 \%$ Tween 20 over a period of $50 \mathrm{~min}$. Bound phage were eluted by incubation of the wells with elution buffer for $15 \mathrm{~min}$ and the collected eluates were neutralised with 2 M Tris. Binding phage were amplified by incubation of the neutralised eluate with $10 \mathrm{ml}$ E.coli TG1 culture $\left(\mathrm{OD}_{600 \mathrm{~nm}}\right.$ of 0.5 ) for $30 \mathrm{mins}$ at $37^{\circ} \mathrm{C}$. Bacteria were pelleted and resuspended in 2xTY and spread on a Nunc Bioassay dish containing TYE agar and $100 \mu \mathrm{g} / \mathrm{ml}$ ampicillin. After o/n incubation at $30^{\circ} \mathrm{C}, 5 \mathrm{ml} 2 \mathrm{xTY}$ containing $100 \mu \mathrm{g} / \mathrm{ml}$ ampicillin and $2 \%$ glucose was added to the plate and the lawn was detached with a sterile scraper.

\subsection{Plaque assay}

The selected peptides were screened directly with the plaque assay. Sufficient cells from the biopanning procedure were grown up and superinfected with M13K07 helper phage for secretion of phage particles with fusion PVIII proteins. Aliquots of these phage were mixed with TG1 culture and Top agar and poured onto LB agar plates. After $5 \mathrm{~h}$ incubation at $37^{\circ} \mathrm{C}$, nitrocellulose filters were applied on the plates and left $\mathrm{o} / \mathrm{n}$ at $37^{\circ} \mathrm{C}$. Filters were blocked for $1 \mathrm{~h}$ with $\mathrm{TBS} / 5 \%$ non- 


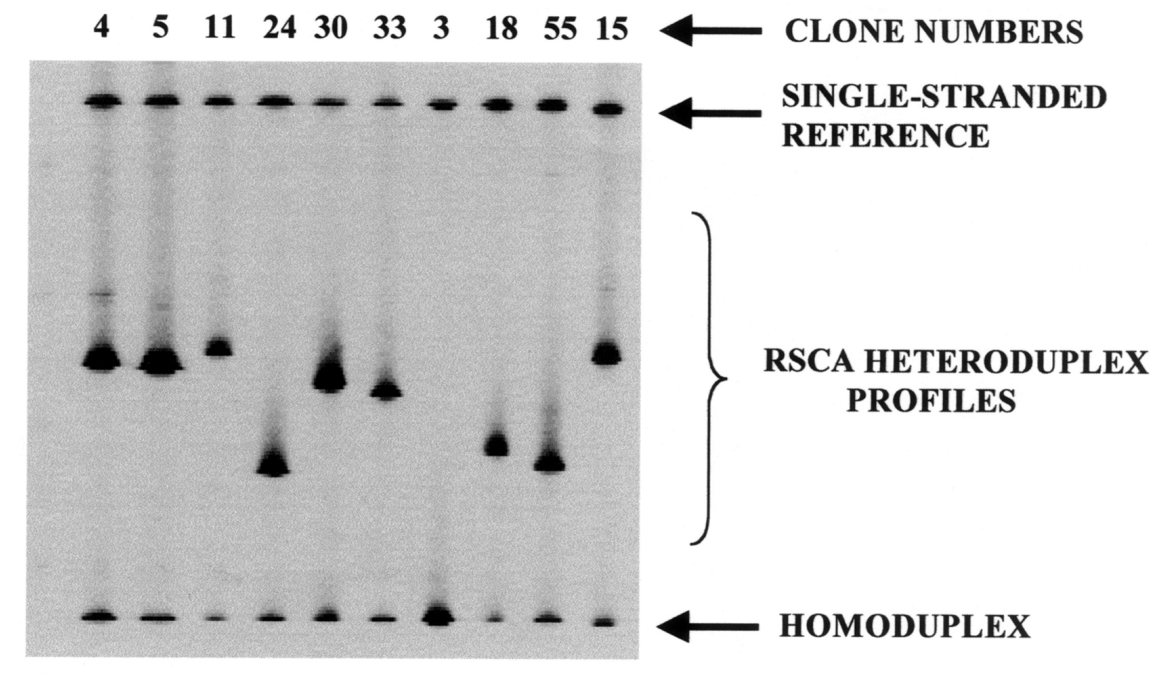

Fig. 1. RSCA analysis of phage clones.

fat dry milk/0.05\% IGEPAL CA-630 and incubated for $2 \mathrm{~h}$ at $\mathrm{rt}$ with mAB $\mathrm{H} 107$ at a final concentration of $1.5 \mu \mathrm{g} / \mathrm{mL}$ in blocking solution and washed 3 times for 5 min each wash in TBS/0.05\% IGEPAL CA-630. Filters were incubated for $1 \mathrm{~h}$ at $\mathrm{rt}$ with anti-mouse IgG Ab conjugated to alkaline phosphatase in blocking solution and washed. The reaction was revealed with nitro-blue-tetrazolium and 5-bromo-4-chloro-3indolyl-phosphate.

\subsection{Polymerase chain reaction (PCR) amplification and sequencing of insert region encoding for binding peptides}

Primer sequences for the phagemid pC89 were selected from either side of the insert region which is located in the coding region for the amino terminus of the PVIII protein. PCR was performed in a Techne PHC-3 Thermal cycler followed by characterisation of the amplified products by agarose gel electrophoresis with a $2 \%$ agarose gel and visualised by ethidium bromide staining. The nucleotide sequence of the insert region was determined with an ABI 373 automated DNA sequencer and both strands were sequenced to ensure accuracy of the sequencing data.

\subsection{Reference strand mediated conformation analysis (RSCA)}

RSCA allows for differentiation between DNA duplexes as such duplexes formed between the reference strand and sample PCR products have a different con- formation and electrophoretic mobility for each sample. Such differences allow for ease of differentiation between peptide binders. For creation of a labelled reference strand, PCR was performed on the phagemid DNA of a previously chosen reference using the same primers as before except the $3^{\prime}$ primer was labelled at the $5^{\prime}$ end with a infra-red label. Duplexes were formed by addition of the reference strand to the sample PCR products gained for the binding clones followed by a denaturation and annealing step. Only duplexes which posses the labelled reference strand were detected using an MWG Biotech Lichor DNA analyser.

\section{Results}

To test RSCA as a means of discriminating between phage clones, 10 binding clones were utilised to check RSCA ability to differentiate between clones which have sequence variations and those which are identical. Clone 3 was arbitrarily chosen for creation of the FLR.

RSCA of the DNA heteroduplexes revealed obvious migration differences between clones 24, 30, 33, 3, 18 and 55, however two pairs of heteroduplexes (clones 11, 15 and clones 4, 5) were found to have similar mobilities. On sequencing all of the antibody-reactive clones it was found that clones 11 and 15 were in fact bearing the same DNA sequence and that all the others had different sequences. 
Table 1

DNA and amino acid sequence of the insert in phage isolated by the panning procedure

\begin{tabular}{|c|c|}
\hline Clone & DNA and aa sequence \\
\hline \multirow[t]{2}{*}{4} & TGCCGTGTGGGGTCTTCGTCTT T CTCCTACTGC \\
\hline & $\mathrm{C}$ \\
\hline \multirow[t]{2}{*}{5} & TGCATCCCGCA GTCCTCTTCCT T TTACATCTGC \\
\hline & Q \\
\hline 11 & TGCTAGGTCA GGGGGTCTGCTT T CAGCTTCTGC \\
\hline$\& 15$ & $\mathrm{R}$ \\
\hline \multirow[t]{2}{*}{30} & TGCGGACGTT GGCCCGGCGACT GGCGTGCCTGC \\
\hline & $\mathrm{W}$ \\
\hline \multirow[t]{2}{*}{18} & TGCTACGACGG TTCGTATCGAT GGGCTCCCTGC \\
\hline & $\mathrm{R} \quad \mathrm{W}$ \\
\hline \multirow[t]{2}{*}{55} & TGCTATGATGC TCAGCACCACTGGACTAGATGC \\
\hline & $\begin{array}{lllllllllllllll}\text { Q } & \mathrm{H} & \mathrm{H} & \mathrm{W} & \mathrm{T} & \mathrm{R} & \mathrm{C}\end{array}$ \\
\hline \multirow[t]{2}{*}{3} & TGCTACAACGCCTTTCTTAACT GGGTCCCATGC \\
\hline & $\mathrm{W}$ \\
\hline *24 & GCTGAACGT C AGCCTTATACT C CGTCCAGTGC \\
\hline$* 33$ & GC的TAACG T CATTGCC TAAC C AACACGCTGC \\
\hline \multicolumn{2}{|c|}{$\begin{array}{l}{ }^{*} \text { The aa sequence of the insert region is unable to be deduced from the } \\
\text { DNA sequence as the reading frame is interrupted by a nonsuppressed } \\
\text { stop codon (underlined). It is uncertain, without further investigation, } \\
\text { whether a post-transcriptional frameshift is needed for expression of the } \\
\text { fusion protein or the ribosome reads through the stop codon. It has } \\
\text { been documented in a recent paper that tryptophan (Trp) was inserted in } \\
\text { place of the UGA stop codon in a significant fraction of an expressed } \\
\text { protein [1]. Substitution of aa's such as Trp at the UGA and UAA stop } \\
\text { codon may have occurred in our fusion proteins. }\end{array}$} \\
\hline
\end{tabular}

\section{Conclusions}

Analysis of phage clones by RSCA showed that clones which were identical upon sequencing could be shown as such by RSCA and clones with sequence variations could, in most cases, be identified by their differential mobility rates. Strategies to distinguish poorly resolved clones (such as clones 4 and 5) are presently being devised which will aid in the discrimination of clones which have diverse sequences but similar mobilities.

Currently, multiple clones are sequenced to determine the level of consensus between clones with time being misspent sequencing identical clones. With the use of RSCA it is hoped that such time and materials wastage can be prevented by its potential to facilitate differentiation of clones that are different and those that are identical. RSCA's ability to detect multiple incidences of the same clone will be of value to those who have previously sequenced clones after several rounds of panning to detect multiply represented clones as an indicator of successful panning.

\section{Acknowledgements}

We thank Alessandra Luzzago for providing the phage library and $\mathrm{H} 107 \mathrm{mAb}$ samples. We are grateful to MWG Biotech for the primer label, Clark Stevenson for primer synthesis and Dr Alan Trudgett for use of the Techne PHC-3 Thermal cycler. We also wish to thank Action Cancer (Northern Ireland), The Association for International Cancer Research and the Parkinson's Disease Society (Armagh Branch) for funding this research.

\section{Reference}

[1] MacBeath and Kast, BioTechniques 24 (1998), 789-794. 


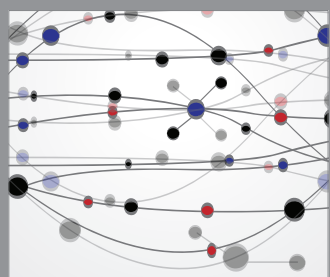

The Scientific World Journal
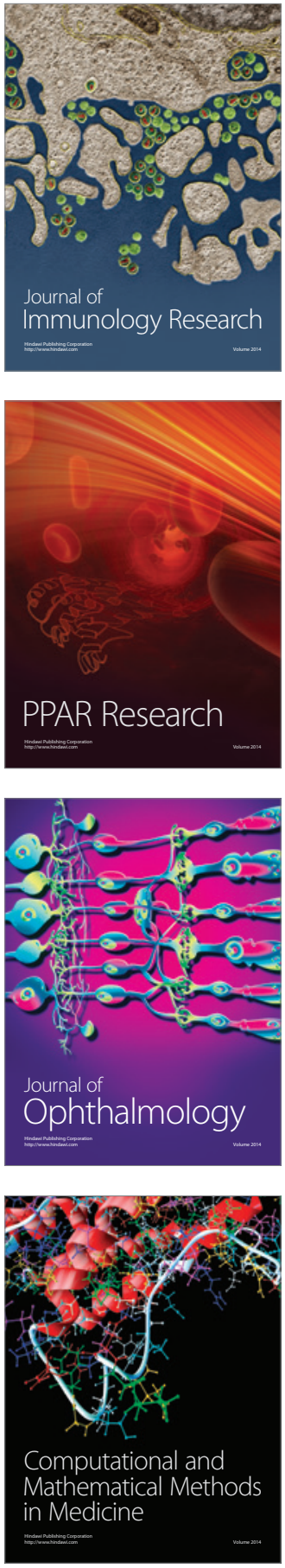

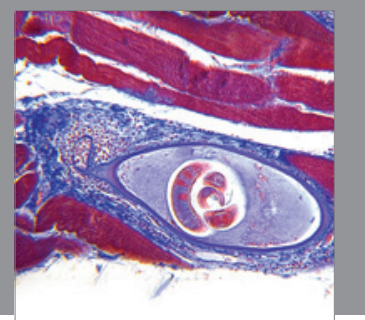

Gastroenterology

Research and Practice
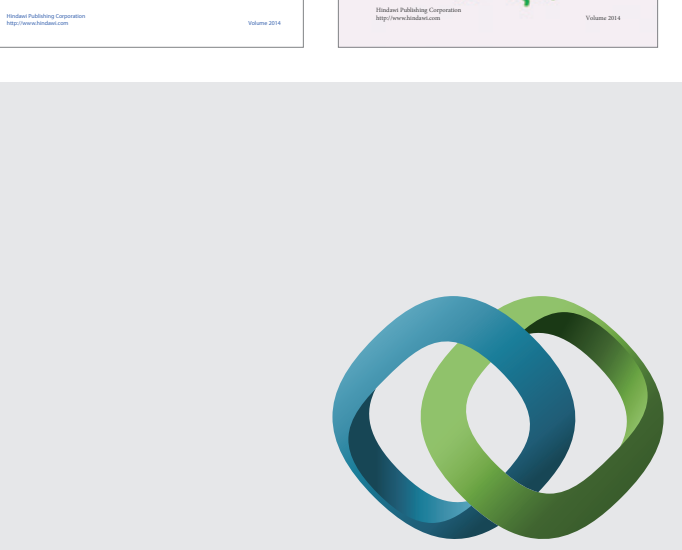

\section{Hindawi}

Submit your manuscripts at

http://www.hindawi.com
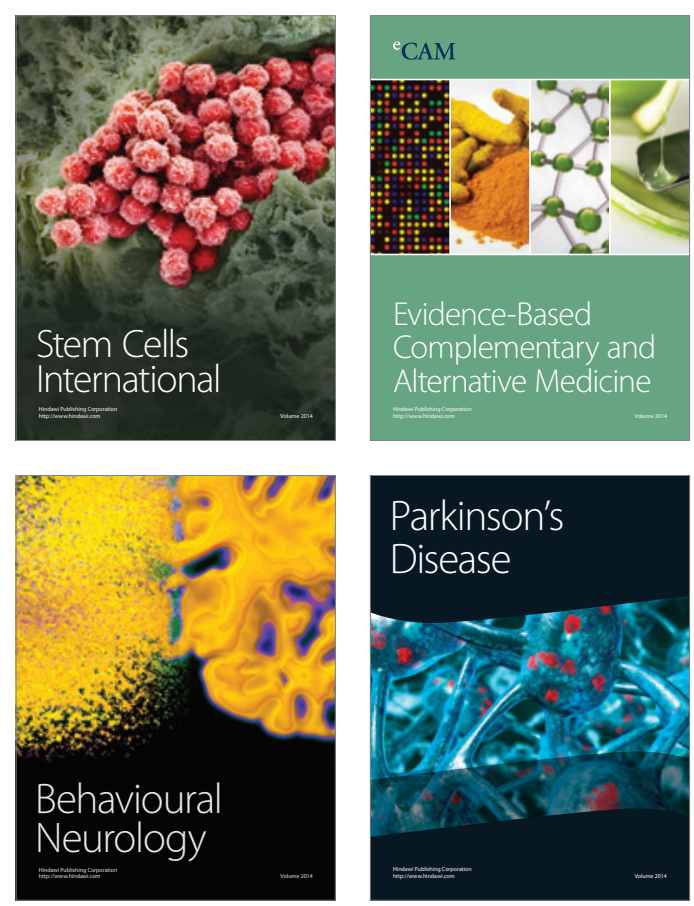

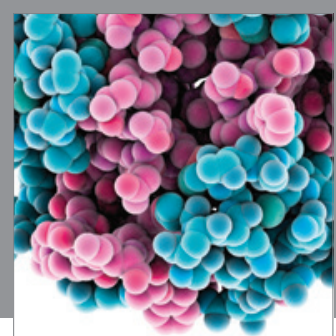

Journal of
Diabetes Research

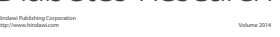

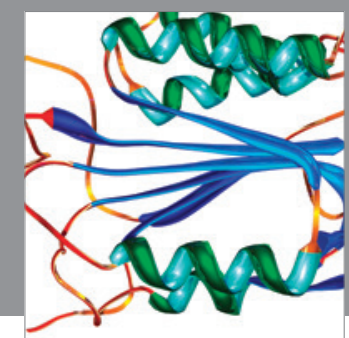

Disease Markers
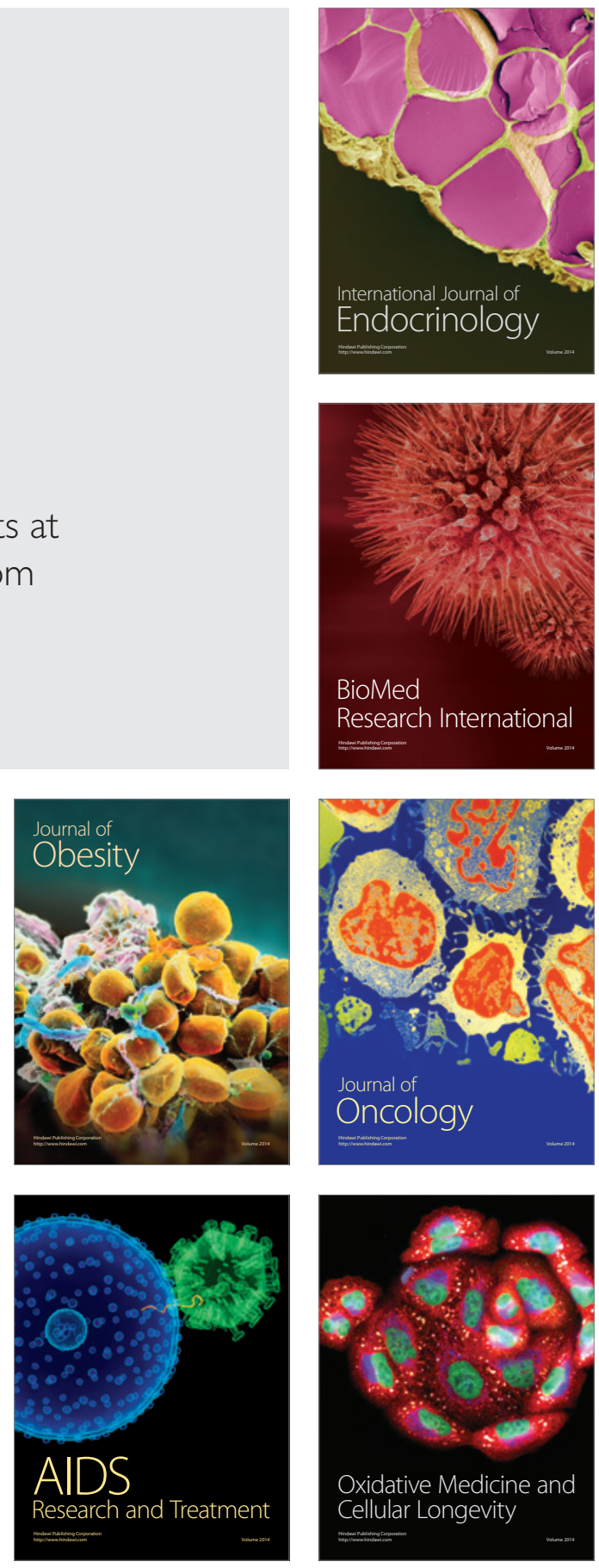